Technological University Dublin

DƯBLIN

ARROW@TU Dublin

2007-01-01

\title{
Investigation of Fast Light in Long Optical Fibers Based on Stimulated Brillouin Scattering
}

\author{
Kai-Uwe Lauterback \\ Deutsche Telekom Fachhochschule Leipzig \\ Thomas Schneider \\ Deutsche Telekom Fachhochschule Leipzig \\ Ronny Henker \\ Deutsche Telekom Fachhochschule Leipzig
}

See next page for additional authors

Follow this and additional works at: https://arrow.tudublin.ie/engschececon

Part of the Electrical and Computer Engineering Commons

\section{Recommended Citation}

Lauterback, K. et al. (2007) Investigation of fast light in long optical fibers based on stimulated Brillouin scattering. CLEO 2007: Conference on Lasers and Electro Optics, pp.1-2. Baltimore, 6-11 May, 2007, doi:10.1109/CLEO.2007.4453658

This Conference Paper is brought to you for free and open access by the School of Electrical and Electronic Engineering at ARROW@TU Dublin. It has been accepted for inclusion in Conference papers by an authorized administrator of ARROW@TU Dublin. For more information, please contact arrow.admin@tudublin.ie, aisling.coyne@tudublin.ie,gerard.connolly@tudublin.ie.

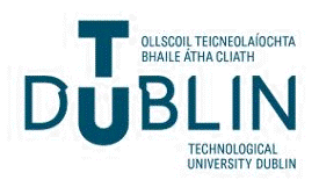




\section{Authors}

Kai-Uwe Lauterback, Thomas Schneider, Ronny Henker, Marcus Junker, Max Ammann, and Andreas Schwarzbacher 


\title{
Investigation of fast light in long optical fibers based on stimulated Brillouin scattering
}

\author{
Kai-Uwe Lauterbach, Thomas Schneider, Ronny Henker, Markus Junker \\ Deutsche Telekom AG, Fachhochschule Leipzig, Gustav-Freytag Str. 43-45, D-04277 Leipzig, Germany \\ kai-uwe.lauterbach@fh-telekom-leipzig.de \\ Max James Ammann, Andreas Thomas Schwarzbacher \\ School of Electronic \& Communications Engineering, Dublin Institute of Technology, Kevin Street Dublin 8, Ireland
}

\begin{abstract}
A simple method to generate a negative time delay in SBS-based fast-light systems using Brillouin gain and loss is shown. We achieved a maximum negative time delay of $32.4 \mathrm{~ns}$ in one long fiber segment.

(C)2007 Optical Society of America

OCIS codes: (999.9999) Fast light; (290.5900) Scattering, stimulated Brillouin
\end{abstract}

Slow and fast light means the control of the velocity of light pulses by light [1]. During the last years a number of experiments and results for slow and fast light in optical fibers based on stimulated Brillouin scattering (SBS) were presented [2-7]. Especially the concept of fast light propagation in optical fibers has attracted much recent interest since it offers the possibility to develop timing tools for all-in-optical signal processing, equalization of distorted optical data streams and fast read out of optical buffers.

The group index of a waveguide $n_{g}$ is described by the equation: $n_{g}=n+\omega d n / d \omega$ [8]. Due to SBS loss it is possible to decrease $n_{g}$ continuously from 1.46 (under normal conditions, refraction index $n$ ) to 0 and also to negative values by changing the term: $\omega d n / d \omega[3]$. Without Brillouin loss the absolute time delay in an optical fiber is given by: $t_{0}=L n_{g} / c_{0}$ and with Brillouin loss: $t_{B}=L n_{g} / c_{0}-L e f f n_{g B} / c_{0}$. Therefore, the relative time delay for slow and fast light systems can be described as: $T_{d}=1 / c_{0}\left(L_{e f f} n_{g B}\right)$.

The measured time delay $T_{d}$ for a standard single mode fiber (SSMF) with a length of $11.8 \mathrm{~km}$ and a Lorentzian distributed Brillouin gain of $-12 \mathrm{~dB}$ (respectively loss) is $-8.4 \mathrm{~ns}$ [2,3]. For very high pump powers the Brillouin gain is $-12 \mathrm{~dB}$ for a $2 \mathrm{~m}$ SSMF. The result is that the group index becomes negative $\left(n_{g B}=-0.7\right)$ this leads to a negative time delay of $T_{d}=-14.4 \mathrm{~ns}$ [3]. Fast light propagation with zero amplification or loss in a $2 \mathrm{~km} \mathrm{SSMF} \mathrm{offers} \mathrm{a} \mathrm{time}$ delay of $-7.3 \mathrm{~ns}$. This result is based on the balanced superposition of a broadened Brillouin gain and natural loss spectrum [6].

Here we investigate light acceleration of up to $32 \mathrm{~ns}$ in a long optical fiber. On the one hand, a long fiber has a high absolute attenuation $(\approx 12 \mathrm{~dB}$ for a $50 \mathrm{~km}$ SSMF). This limits fast light generation since it depends on the Brillouin loss. Together with the fiber loss this would result in a very high attenuation of the probe pulse. On the other hand, the Brillouin threshold is very low for long fibers. A few milliwatt of pump power are sufficient to initiate SBS. Our method is based on an idea which was presented in $[6,7]$.

Figure 1 shows our principle experimental setup. We used a fiber laser followed by a Mach-Zehnder modulator (MZM) for generating the pulse spectrum at a wavelength of $1550 \mathrm{~nm}$. This wavelength is equivalent to the signal frequency $f_{s}$. The pulse generator excites Gaussian pulses with a repetition rate of $1 \mathrm{MHz}$ and a pulse width of $35 \mathrm{~ns}$. These pulses were transmitted over a $50 \mathrm{~km} \mathrm{SSMF}$ and an optical circulator (C), (port 2 to 3); finally detected by a PIN photodiode and evaluated in the time domain by an oscilloscope. From the other side a pump spectrum was coupled into the same SSMF over the circulator (port 1 to 2). Due to this very long SSMF the required pump power can be reduced drastically. For the generation of the Brillouin spectrum, we used two uncorrelated distributed feedback (DFB) laser diodes as pump lasers at $1550 \mathrm{~nm}$ (equal to pump frequency $f_{P}$ ). Each pump laser engendered a Brillouin gain spectrum at $f_{P}-f_{B}$ and a Brillouin loss spectrum at $f_{P}+f_{B}$, with $f_{B}$ as the natural Brillouin shift in the fiber. In a SSMF $f_{B}$ is approximately $11 \mathrm{GHz}$. In our experimental setup the pump laser 1 generated the Brillouin gain spectrum, with the line centre Brillouin gain $g_{B}$ at the signal frequency $f_{S}$. Therefore, it is necessary that $f_{S}=f_{P I^{-}}$ $f_{B}$. We broadened the Brillouin gain spectrum by a direct modulation of pump laser 1 with a noise source. Furthermore, pump laser 2 generates the maximum Brillouin loss $-g_{B}$ at the signal frequency $f_{S}$ according to the relationship $f_{S}=f_{P 2}+f_{B}$. Under the condition that $f_{S}=f_{P 1}-f_{B}=f_{P 2}+f_{B}$ the signal frequency as well as the Brillouin gain and loss superimpose. The power of Brillouin gain and loss is adjustable by an erbium-doped fiber amplifier (EDFA) and a tunable optical attenuator (TOA). The result is an added tunable Brillouin loss within a tunable broadened Brillouin gain at the signal frequency. The $-3 \mathrm{~dB}$ bandwidth of the broadened gain spectrum is $160 \mathrm{MHz}$. 


\section{JWA48.pdf}

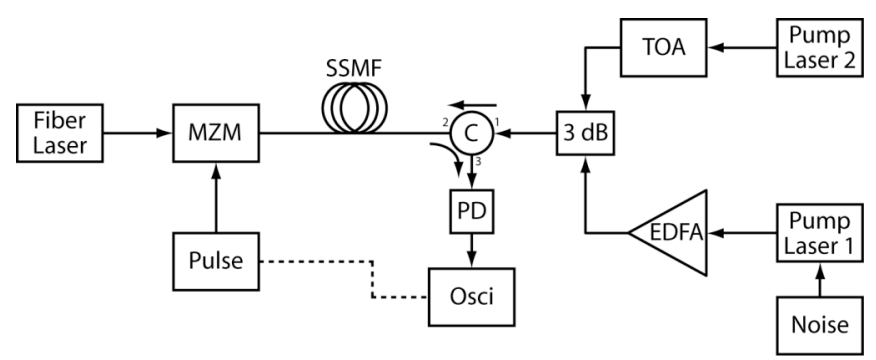

Figure 1: Experimental setup. MZM: Mach-Zehnder modulator; Pulse: pulse generator; SSMF: standard single mode fiber; C: circulator; PD: PIN-photodiode; Osci: oscilloscope; $3 \mathrm{~dB}: 3 \mathrm{~dB}$ coupler; TOA: tunable optical attenuator; EDFA: erbium-doped fiber amplifier; Noise: noise generator

Figure 2 shows the negative time delay as a function of the power of pump laser 2 for different pump powers $(5.5 \mathrm{~mW}, 8.7 \mathrm{~mW}, 13.8 \mathrm{~mW}$ and $21.9 \mathrm{~mW})$ of laser 1 . The specified power values in Figure 2 are measured at the port 2 of the circulator. The measured time delay is specified as the time difference between the transmission time under the condition of stimulated Brillouin scattering (SBS) and without SBS; $T_{d}=t_{B}-t_{0}$, where $t_{0}=L n_{\mathrm{g}} / c$. For increasing pump power of laser 2 the negative time delay increases. For moderate pump powers $(5.5 \mathrm{~mW}, 8.7 \mathrm{~mW})$ of laser 1 the time delay as a function of pump power of laser 1 is linearly. For higher pump powers of laser 1 we allready start with a negative time delay. This is due to the case that for large gains $G=g_{0} I_{P} L_{e f f}$ the time delay becomes negative even for a Brillouin gain [5].

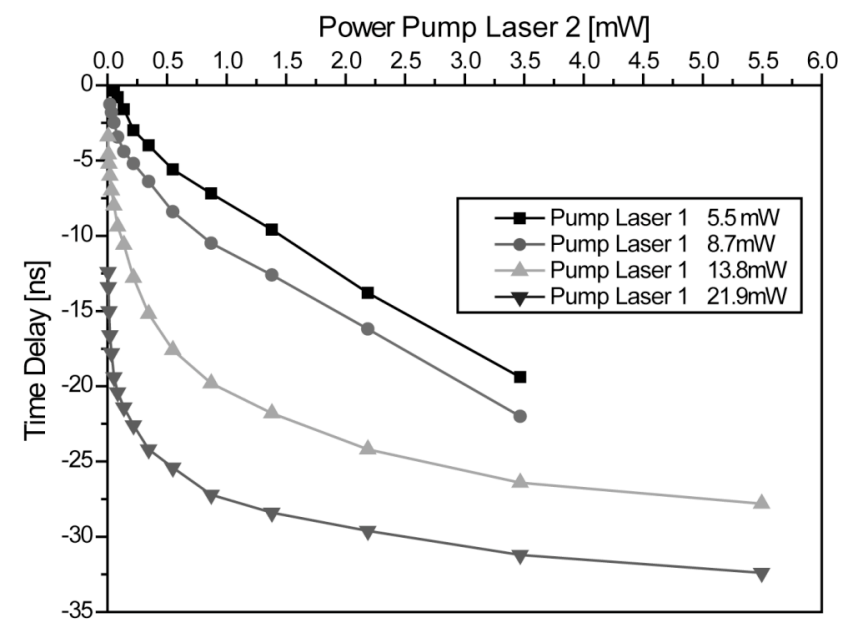

Figure 2: Negative time delay against Brillouin loss pump power for different gain pump powers

In conclusion we have shown that by the superposition of Brillouin gain and loss fast light in optical fibers can be achieved. With this method we obtain a maximum negative time delay of $32.4 \mathrm{~ns}$ in a $50 \mathrm{~km}$ SSMF.

We gratefully acknowledge the help of J. Klinger of Fachhochschule Leipzig.

\section{References}

[1] R. W. Boyd, N. Lepeshkin, A. Schweinsberger, and P. Zerom, "Fundamentals and Applications of Slow and Fast Light," International Quantum Electronics Conference 2005, 1187-1188 (2005).

[2] M. González-Herráez, K. Y. Song, and L. Thévenaz, "Optically controlled slow and fast light in optical fibers using stimulated Brillouin scattering," Appl. Phys. Lett. 87, 081113 (2005).

[3] K. Y. Song, M. González-Herráez, and L. Thévenaz, "Observation of pulse delaying and advancement in optical fibers using stimulated Brillouin scattering," Opt. Express 13, 82-88 (2005).

[4] Y. Okawachi, M. S. Bigelow, J. E. Sharping, et al., “Tunable All-Optical Delays via Brillouin Slow Light in an Optical Fiber,” Phys. Rev. Lett. 94, 153902 (2005).

[5] Z. Zhu, D. J. Gauthier, Y. Okawachi, et al., "Numerical study of slow light via stimulated Brillouin scattering in optical fibers," QELS '05, 844-846 (2005).

[6] S. Chin, M. González-Herraez, and L. Thévenaz, "Zero-gain slow \& fast light propagation in an optical fiber," Opt. Express 14, 10684 (2006).

[7] Th. Schneider, M. Junker, K.-U. Lauterbach, "Time delay enhancement in stimulated Brillouin scattering based slow light systems," Opt. Lett. 32, (2007).

[8] Th. Schneider, Nonlinear Optics in Telecommunications (Springer-Verlag, 2004). 